\title{
EVALUATION OF OFF-LINE AUTOMATED INTIMA-MEDIA THICKNESS DETECTION OF THE COMMON CAROTID ARTERY BASED ON M-LINE SIGNAL PROCESSING
}

\author{
Christine Willekes, ${ }^{*}$ Arnold P. G. Hoeks, ${ }^{\dagger}$ Michiel L. Bots, ${ }^{\dagger}$ Peter J. Brands, ${ }^{\dagger}$ \\ JeAn M. Willigers, ${ }^{\dagger}$ and Robert S. Reneman* \\ Departments of *Physiology and ${ }^{\dagger}$ Biophysics, Cardiovascular Research Institute Maastricht, Maastricht University, \\ Maastricht, and Department of Epidemiology \& Biostatistics, Erasmus University Medical School Rotterdam, \\ Rotterdam, The Netherlands
}

(Received 5 May 1998; in final form 12 August 1998)

\begin{abstract}
Intima-media thickness (IMT) measurements have gained increasing attention, because IMT is assumed to represent the endothelial adaptive response to physiological and pathophysiological processes. The main aim of the present study was to assess the intrasubject intrasession variability of a new off-line automated radio frequency (RF) IMT method in comparison with an already established off-line manual B-mode IMT method. IMT also was assessed by means of an on-line manual B-mode and an on-line manual RF IMT method. We investigated posterior wall IMT 0-1 cm proximal to the bulb in both common carotid arteries of 16 young (20-31 y; mean 25 y) female and male and 13 elderly (51-65 y; mean 56 y) female volunteers. Two commercially available ultrasound devices (Pie Medical Scanner 200 and Ultramark 9) were used to assess the effects of signal processing on the off-line automated RF IMT method. Intrasubject intrasession variability was determined using the standard deviation to evaluate and compare the various methods. Spearman rank correlation coefficients and Bland and Altman bias and limits of agreement were calculated to objectivate the comparability between the various methods. Intrasubject intrasession variation of IMT estimates was not statistically different between any of the methods. We observed a good comparability between the commonly used off-line manual B-mode IMT method and the off-line automated RF IMT method at the level of the common carotid artery. (C) 1998 World Federation for Ultrasound in Medicine \& Biology.
\end{abstract}

Key Words: Ultrasound, Intima-media thickness, Carotid artery, Automated wall thickness detection, M-mode data processing.

\section{INTRODUCTION}

In the past decade, the intima-media thickness (IMT) of the artery wall has gained increasing attention, because it is assumed to represent the endothelial adaptive response to physiological and pathophysiological processes, such as those seen in ageing and hypertension (O'Leary et al. 1996; Persson et al. 1994; Salonen and Salonen 1990, 1991; Wong et al. 1993). A variety of IMT measurement protocols, based on different ultrasound methods, have been developed and improved in order to obtain reliable and reproducible results (Hoeks et al. 1997; Persson et al. 1992; Pignoli et al. 1986; Riley et al. 1992; Salonen et al.

Dr. Bots' present address is: Julius Center for Patient Oriented Research, University Utrecht, Utrecht, The Netherlands.

Address correspondence to: Arnold P. G. Hoeks, Department of Biophysics, CARIM, Maastricht University, P.O. Box 616, $6200 \mathrm{MD}$ Maastricht, The Netherlands. E-mail: A.Hoeks@BF.Unimaas.nl
1991; Selzer et al. 1994; Wendelhag et al. 1997; Wong et al. 1993).

In 1984, it was shown, using calipers, that in vitro artery wall thickness can be assessed reliably and accurately by B-mode imaging, and the results obtained correlated well with histologic examinations (Pignoli 1984). In 1986, it was demonstrated that IMT also can be assessed in vivo, and that it indeed represents the true intima-media complex as confirmed by histologic recognition (Pignoli et al. 1986). The method, however, shows a considerable variation. This is partly attributed to the limited axial resolution and a relatively poor magnification of the B-mode imagers at that time. Thanks to technical improvements in displaying the received B-mode signal and better zoom possibilities, the current ultrasound devices are capable of discerning intima-media complexes more accurately with a precision of $0.1 \mathrm{~mm}$ (Bots et al. 1994). 
One of the other methods to measure IMT uses automated digitization of the B-mode image, after capturing it in a preselected phase of the cardiac cycle with a frame grabber, and subsequent off-line analysis (Bond et al. 1991; Riley et al. 1992). Aside from the high user interactivity, the speckle pattern and the signal preprocessing in the ultrasound scanner system prior to video presentation are important restrictions of this method. To delineate the different boundaries of the intima and media, markers have to be placed manually on the video image (Bond et al. 1991; Wendelhag et al. 1991). This is time consuming and introduced some subjectivity, dependent on the reflectivity of the structures and the gain setting and signal compression characteristics of the ultrasound system. The process was improved and made quicker by automated interpolation of the intima-media complex after simple identification by the observer (Chambless et al. 1996; Riley et al. 1996). This improved the reproducibility of the procedure and rendered it more user independent. Additionally, automated spatial-averaging procedures circumvented the dependence of the outcome on the threshold level by adjusting the threshold according to the local characteristics of the nonlinear B-mode signal (Selzer et al. 1994; Wendelhag et al. 1997). The off-line manual as well as off-line automated B-mode IMT methods provide a mean IMT over a length of $10 \mathrm{~mm}$.

The B- and M-mode signals displayed on the ultrasound monitor are derived from the received raw radio frequency (RF) signals. These RF signals have, among others, been used to assess artery wall displacement with a wall track system (WTS) (Hoeks et al. 1990). The RF signals also can be used to measure IMT locally by manually placing markers on amplitude signals of the blood-intima interface and the media-adventitia interface as induced by the end-diastolic RF line. The off-line automated RF IMT measurement method uses temporal averaging of the RF signals received over the cardiac cycle, rather than spatial averaging of the echo signal (Hoeks et al. 1997) to reduce the incidental influence of speckle. Considering the median value of the IMT estimates, obtained over a few subsequent cardiac cycles, will improve the precision of the IMT estimate. The automated procedure is more time efficient than the previously mentioned manual B-mode methods and rather user independent. It calculates mean IMT locally over a cardiac cycle instead of mean IMT over a 10-mm segment at end-diastole. The reproducibility of this method is good, as published previously (Hoeks et al. 1997).

The aim of the present study was to evaluate the intrasubject intrasession variability and comparability of the various procedures to estimate IMT (manual on-line and automated off-line B-mode and RF processing), us- ing two different commercially available ultrasound systems (Ultramark 9 and Pie Medical Scanner 200). No on-line manual B-mode IMT measurements were performed on the Pie Medical Scanner 200, because of the absence of adequate zoom magnification. The on-line manual RF IMT method was performed only on the first RF line of the Pie Medical Scanner 200, because of its use in combination with the WTS. To investigate whether our RF IMT method measured the same as the B-mode IMT method, the results of both methods were compared. The measurements were performed on the left and right common carotid arteries of volunteers of different ages.

\section{MATERIAL AND METHODS}

\section{Subjects}

The study was performed on 16 presumed healthy male and female volunteers (20-31 y; mean $25 \mathrm{y}$ ), equally divided over both genders, and an additional group of 13 presumed healthy females (51-62 y; mean $56 \mathrm{y})$. Both young and elderly volunteers were used to obtain an adequate range of IMT values, as ageing induces a physiological and consistent increase in IMT. The study was approved by the joint Medical Ethics Committee of the Maastricht University and the Academic Hospital Maastricht. All volunteers gave written consent after receiving detailed information before entering the study.

\section{Ultrasound systems}

The IMT measurements were performed using two different commercially available ultrasound devices. The Pie Medical Scanner 200 (Pie Medical, Maastricht, The Netherlands) has a linear array transducer of 7.5 MHz. The measured pulse length at $-6 \mathrm{~dB}$ as received from a tissue target at a distance of $20 \mathrm{~mm}$ is $0.35 \mu \mathrm{s}$, corresponding to an axial resolution of approximately 0.26 $\mathrm{mm}$. This scanner has an electrocardiographic (ECG) modality to start data collection at the end of diastole. The Ultramark 9 (ATL, Bothell, WA, USA) has a linear array transducer of $6-10 \mathrm{MHz}$. The measured pulse length at $-6 \mathrm{~dB}$ is $0.31 \mu \mathrm{s}$, corresponding to an axial resolution of approximately $0.22 \mathrm{~mm}$. The device contains a cine-loop function to review stored images over one to two cardiac cycles together with the recorded ECG trace so that the selection of a specific phase of the cardiac cycle can be performed manually. A built-in super VHS video recorder allows storage of the obtained images for off-line assessment of IMT, according to spatial B-mode averaging.

\section{Intima-media thickness measurement protocol}

We measured the IMT of both the left and the right common carotid arteries at $0-1 \mathrm{~cm}$ proximal to the bulb 


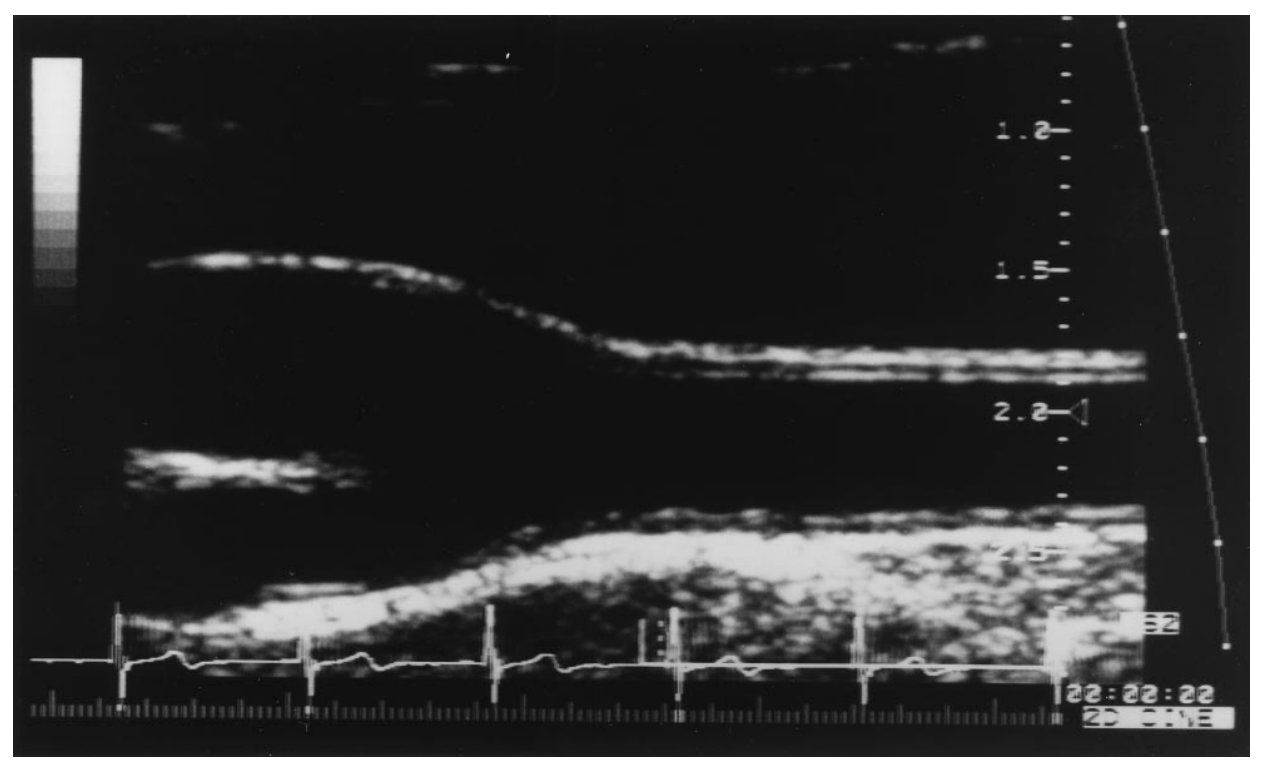

Fig. 1. B-mode photography of the right common carotid artery of a 52-y-old healthy volunteer obtained using the Ultramark 9. The intima-media complex clearly is visible at the posterior and anterior common carotid artery wall over a distance of at least $1 \mathrm{~cm}$. At the left, the widening of the bulb is used as a landmark for intima-media thickness measurements.

in the plane of the bifurcation (Fig. 1). Only the posterior wall was investigated, because here the reflections from the blood-intima and media-adventitia transitions are distinctly visible, whereas, at the anterior wall, the trailing edge of the adventitia reflections may obscure the media and intima signal. We compared five different methods in use to assess IMT. All measurements were performed by one single investigator (C.W.). The reading of the stored end-diastolic B-mode images was performed in Rotterdam by an experienced ultrasonographer.

\section{Image analysis}

Off-line automated RF technique. First, we measured local IMT 0 and $1 \mathrm{~cm}$ proximal to the bulb by means of the off-line automated RF IMT method based on M-line signal processing as described in detail previously (Hoeks et al. 1997), using the Ultramark 9. The off-line automated RF IMT method was based on the following procedure. A B-mode image was obtained of the common carotid artery in a longitudinal plane, after which an M-line was positioned perpendicular to the posterior wall, showing a clear intima-media complex. Subsequently, the system was switched to M-mode and, after ECG triggering, RF signal collection started in the data acquisition memory of the WTS at a sample frequency of $20 \mathrm{MHz}$ and an M-line update frequency of $400 \mathrm{~Hz}$ for a period of $4 \mathrm{~s}$. Consecutively, the first RF line was displayed on the computer monitor, allowing identification and selection of a window of $3 \mathrm{~mm}$, covering the posterior intima-media complex. Data from this window over time were stored on hard disk for additional off-line processing. At the end of the measurement protocol, the recorded files were processed using the wall thickness program. The system settings were held constant, with a threshold of the derivative of the amplitude at 0.02 (2\% of peak amplitude adventita per sample distance; approximately $35 \mu \mathrm{m}$ ). The RF signals were aligned in phase prior to averaging based on the detected displacement between observations, as provided with the WTS algorithm. Averaging over the systolic phase of a heart beat enhanced signals from wall interfaces exhibiting similar displacements between observations and smoothed secondary echo signals (e.g., reverberations) with a different displacement behavior. For each heart beat, the derivative of the averaged amplitude distribution was considered with respect to depth to distinguish wall signals from other signals. Only the leading edge of distinct echoes exceeding the threshold for the derivative was used to identify relevant regions. The position where the instantaneous amplitude was halfway in the amplitude range within the region was considered the location of the intima edge. This detection procedure was repeated for the next region (possibly the media-adventitia edge), where the derivative exceeded the threshold. Each heart beat within a recording resulted in an estimate of IMT. The ratio of intima to adventitia was at least 1.0, to suppress detection of false edges. The 
resolution for wall thickness detection was approximately $40 \mu \mathrm{m}$ (Hoeks et al. 1997). The lower limit for intima-media wall thickness was determined by the axial resolution of the ultrasound device used. Measurements were repeated three times at both 0 and $1 \mathrm{~cm}$ proximal to the bulb in the common carotid artery. The average of the median values obtained at both locations was taken as a representative IMT value.

Second, we used the Pie Medical Scanner 200 in combination with the off-line automated RF IMT method to evaluate the effect of system characteristics on the estimated IMT. The Pie Medical Scanner 200 uses a sample frequency of $22.1 \mathrm{MHz}$ and an M-line update frequency of $500 \mathrm{~Hz}$.

Off-line manual B-mode technique. Third, we used the off-line manual B-mode approach. The B-mode images obtained with the Ultramark 9 were frozen at the $\mathrm{R}$ wave of the ECG ( end-diastolic phase). Clearly visible intima-media complexes of the common carotid artery more than $1 \mathrm{~cm}$ from the bulb were selected. The frozen images were expanded maximally to attain maximum detail (zoom), stored on super VHS video tape, and sent to an analysis center for additional analysis. The video tapes were read according to a standardized procedure described in detail previously (Bots et al. 1994). Briefly, the frozen images from the video tape were digitized and displayed on the screen of a personal computer using additional dedicated software (Wendelhag et al. 1991). The lumen-adventitia and media-adventitia interfaces of the distal common carotid were marked with a cursor over a length of $10 \mathrm{~mm}$. The beginning of the dilatation of the common carotid artery (bulb) served as a reference point for the start of the measurements. The mean value of the end-diastolic IMT of the corresponding segment was calculated. This procedure was repeated for three different images obtained on both sides, and the average of the three measurements was taken as a representative for comparison.

On-line manual B-mode technique. Fourth, the same Ultramark $9 \mathrm{~B}$-mode image was used to assess IMT on-line with the aid of callipers of the Ultramark 9, which can be moved with a precision of $0.1 \mathrm{~mm}$. Each image was analysed instantaneously at two places in the common carotid artery $(0$ and $1 \mathrm{~cm}$ proximal to the widening of the bulb) at consistent gain and zoom settings. Three different measurements were performed at each site, and the average of both locations was taken as the reading.

On-line manual RF technique. Fifth, we used the Pie Medical Scanner 200 manually to place sample volumes on the first RF line obtained at end-diastole. This end-diastolic RF line was displayed on the computer

\section{RF-line WTS Pie Medical Scanner 200}

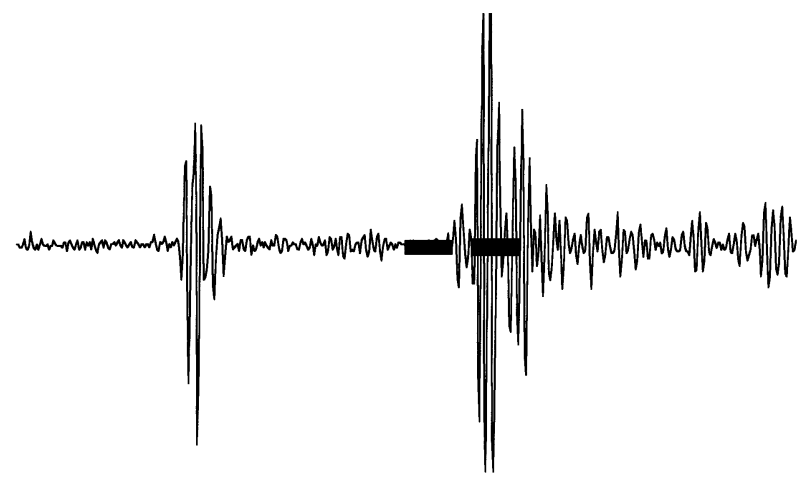

\section{= sample volume}

Fig. 2. Sample volumes (black boxes) positioned on the first radio frequeny (RF) line received from the Pie Medical Scanner 200 at end-diastole are used to estimate intima-media thickness. The right side of the left sample volume is placed in the beginning of the lumen-intima reflection, and the left side of the right samle volume is placed in the beginning of the media-adventitia transition of the posterior wall. The distance between both edges of the sample volumes is presented with a precision of two decimal places. WTS $=$ wall track system.

monitor for assessment of local artery wall movement, according to the method of Hoeks et al. (1990). The placement of sample volumes at the posterior bloodintima interface and media-adventitia interface (Fig. 2) mimics partially the off-line automated RF IMT protocol, albeit that only the end-diastolic artery wall thickness is considered and that speckle interference on the raw $\mathrm{RF}$ signal is not reduced by averaging over the cardiac cycle. Data are presented (in $\mathrm{mm}$ ) on the computer monitor with accuracy to two decimal places, whereas the RF line is discretized in steps of $35 \mu \mathrm{m}$. For documentation, a printout of the screen was made. Three measurements were performed at the same locations in the common carotid artery, as mentioned in the automated RF method, and again the average of both locations was used as representative of each side in the artery.

Two different ultrasound devices were used in this protocol because we wanted to establish the influence of ultrasound signal processing on the outcome of the automated RF IMT technique. Also, the zoom capacities of the Pie Medical Scanner 200 were limited such that we were unable to attain proper end-diastolic B-mode images for analysis by the on-line and off-line B-mode techniques. Finally, we already had used the Pie Medical Scanner 200 in combination with the WTS and wanted to establish the possibility of assessing IMT based solely on 
Table 1. Intrasubject intrasession intima-media thickness estimates

\begin{tabular}{lccccc}
\hline & \multicolumn{2}{c}{ Young } & & \multicolumn{2}{c}{ Elderly } \\
\cline { 2 - 3 } \cline { 6 - 6 } \multicolumn{1}{c}{ Method } & Left & Right & & Left & Right \\
\hline On-line B-mode & $483(27)$ & $494(31)$ & & $714(43)$ & $708(64)$ \\
Off-line B-mode & $492(30)$ & $522(26)$ & & $704(35)$ & $693(37)$ \\
On-line RF line & $494(30)$ & $514(25)$ & & $682(46)$ & $669(44)$ \\
Off-line RF UM 9 & $494(45)$ & $513(64)$ & & $713(46)$ & $733(69)$ \\
Off-line RF Pie & $523(46)$ & $549(42)$ & & $739(74)$ & $730(61)$ \\
\hline
\end{tabular}

Mean intima-media thickness (IMT) \pm SD is given in $\mu \mathrm{m}$. Wilcoxon nonparametric signed-rank tests revealed no significant differences between left or right carotid artery or any IMT method.

the end-diastolic RF line presented on the monitor during the WTS measurement protocol.

\section{Statistical analysis}

Difference in intramethod intrasession variability was assessed by means of the Wilcoxon nonparametric signed-rank test for statistical significant difference in standard deviations (SDs) between the various methods (Table 1). SPSS software packages were used to calculate the Spearman rank correlation coefficient between various methods. We determined the corresponding measurement agreement by assessing the bias (mean differ-

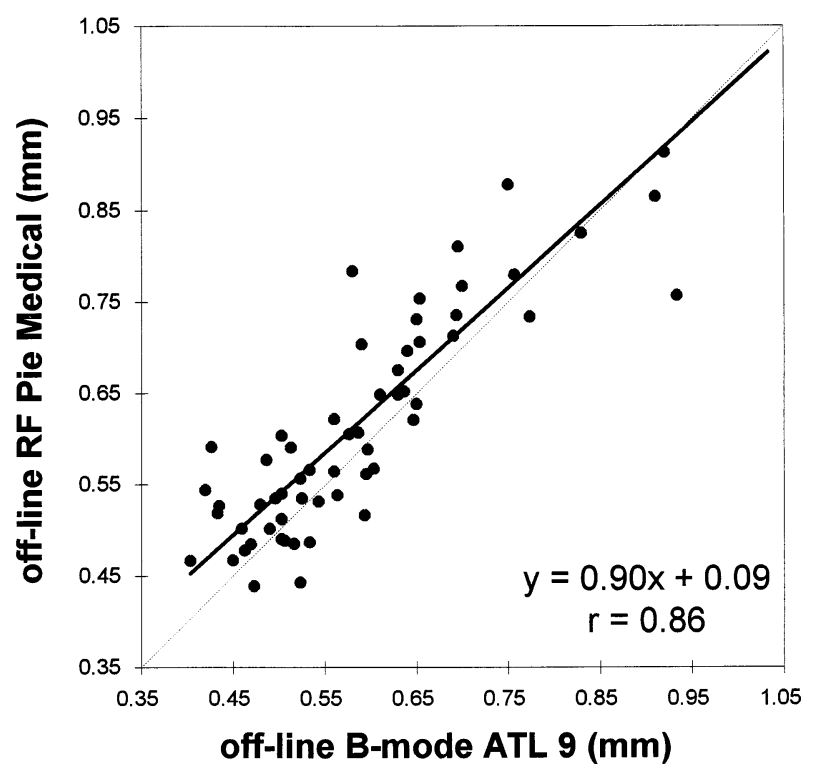

Fig. 3. Intima-media thickness (IMT) results obtained with the off-line automated radio frequency (RF) IMT method using the Pie Medical Scanner 200 compared with the off-line manual B-mode ATL IMT results. The regression line is displayed, as well as its equation and the $r$ value of the Spearman rank correlation coefficient. The dotted line represents the line of identity.

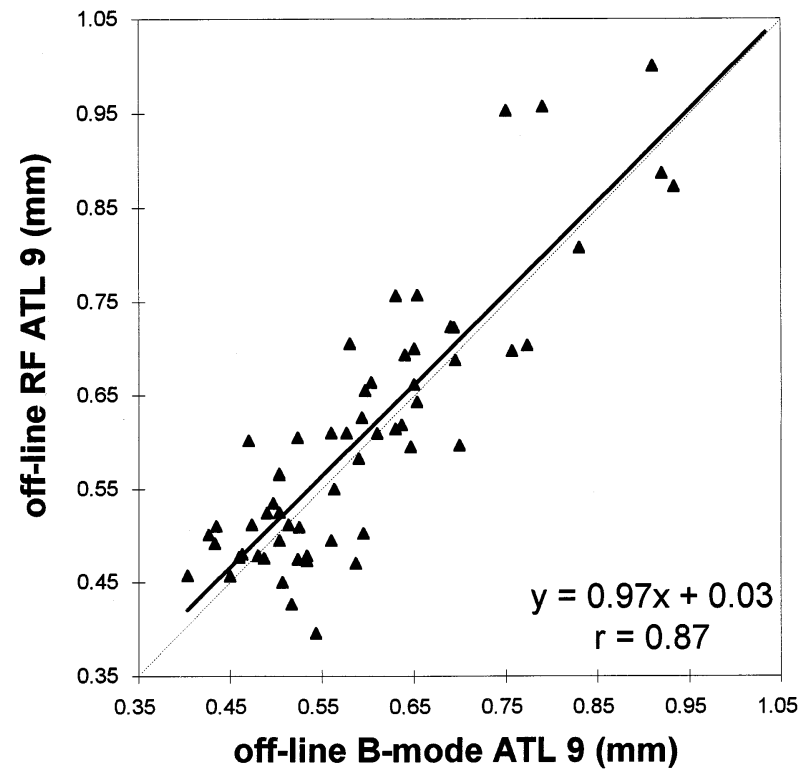

Fig. 4. Intima-media thickness (IMT) results obtained with the off-line automated radio frequency (RF) IMT method using the Ultramark 9 compared with the off-line manual B-mode IMT results. The regression line is displayed, as well as its equation and the $r$ value of the Spearman rank correlation coefficient. The dotted line represents the line of identity.

ence [d]) and limits of agreement $(=\mathrm{d} \pm 2 \mathrm{SD})$ as described by Bland and Altman (1986). After acknowledging the normal distribution of mean differences in IMT between left and right common carotid artery with each method and the relative small bias between both sites (minimum $-31 \mu \mathrm{m}$; maximum $11 \mu \mathrm{m}$ : not statistically significantly different), IMT measurements of both common carotid arteries were included as independent values in the statistical analysis for each method. In case of one missing value, the average of the remaining two values was taken. On one occasion, two values were missing, when the automated IMT method was used. The one remaining value was taken as the subject's reading.

\section{RESULTS}

The intrasubject intrasession variation of IMT estimates was not statistically significantly different between the various methods (Table 1). No significant differences between left and right common carotid arteries were observed by means of paired $t$-tests using SPSS software packages. The on-line manual placing of calipers on the enlarged B-mode image of the artery gave as good results as any of the other methods (SD range for young subjects: $27-64 \mu \mathrm{m}$ ).

The correlations between the off-line manual Bmode IMT method and our off-line automated RF IMT method for both systems are shown in Figs. 3 and 4. The 


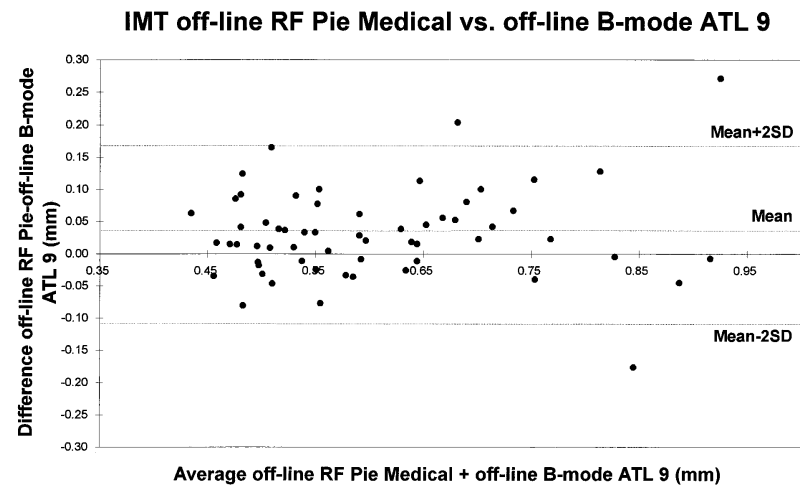

Fig. 5. Bland-Altman comparison between the off-line automated radio frequency (RF) intima-media thickness (IMT) values obtained with the Pie Medical Scanner 200 and the on-line manual B-mode IMT values. Depicted are the difference versus mean of both methods.

correlation coefficients are satisfactory and highly significant $(r=0.86$ and 0.87 , respectively; both $p<$ 0.0001 ). The measurement agreements as depicted in Figs. 5 and 6 for either comparison are good, with a bias of $31 \mu \mathrm{m}$ (off-line automated RF IMT Pie Medical minus off-line manual B-mode IMT Ultramark 9) and 13 $\mu \mathrm{m}$ (off-line automated RF IMT Ultramark 9 minus off-line manual B-mode IMT Ultramark 9) and with limits of agreement of $-109 \mu \mathrm{m}$ to $+171 \mu \mathrm{m}$ and -125 $\mu \mathrm{m}$ to $+151 \mu \mathrm{m}$, respectively. In nearly all cases $(98 \%$ off-line automated RF method; $92 \%$ off-line manual B-mode IMT method), values were obtained. Both the on-line manual RF and B-mode IMT methods showed a fair association with the off-line manual B-mode IMT method ( $r=0.83$ and 0.90 , Figs. 7 and 8, respectively). This is confirmed by the mean differences $(-12 \mu \mathrm{m}$ and

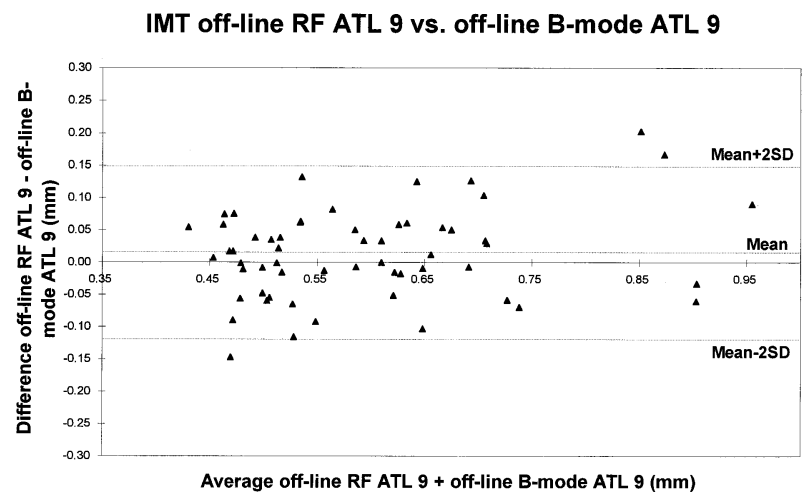

Fig. 6. Bland-Altman comparison between the off-line automated radio frequency (RF) intima-media thickness (IMT) values obtained with the Ultramark 9 and the on-line manual B-mode IMT values. Depicted are the difference versus mean of both methods.

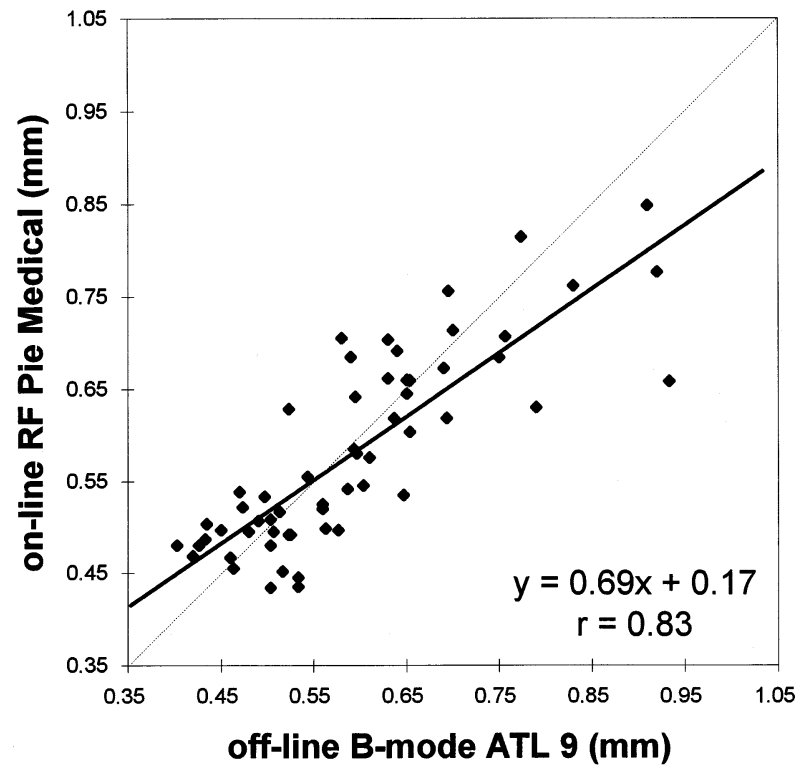

Fig. 7. Intima-media thickness (IMT) results obtained with the off-line manual B-mode IMT method as compared with the on-line manual RF IMT method. The regression line is displayed, as well as its equation and the $r$ value of the Spearman rank correlation coefficient. The dotted line represents the line of identity.

$-5 \mu \mathrm{m}$, respectively) and limits of agreement as seen in the corresponding Bland-Altman figures $(-152 \mu \mathrm{m}$ to $+128 \mu \mathrm{m}$ and $-119 \mu \mathrm{m}$ to $+109 \mu \mathrm{m}$, Figs. 9 and 10, respectively).

\section{DISCUSSION}

In the present study, we determined the intrasubject intrasession variability and comparability of various manual and automated B-mode and RF processing methods used to estimate common carotid IMT. We observed a good correlation and measurement agreement between the formerly established and commonly used off-line manual B-mode IMT method (Bond et al. 1991; Bots et al. 1994; Chambless et al. 1996; Riley et al. 1992; Selzer et al. 1994; Wendelhag et al. 1991; Wong et al. 1993) and our recently developed off-line automated RF IMT method based on M-line signal processing (Hoeks et al. 1997).

The intrasubject intrasession variability is acceptable and comparable in all methods used (Table 1). The manual B-mode method performs equally well as the off-line methods, which might lead to the conclusion to skip the latter methods in favor of the former when considering time efficiency. One should realize, however, that the manual B-mode method is subjective in nature. It is impossible to perform IMT measurements in a blinded way with this method, making it less suitable 


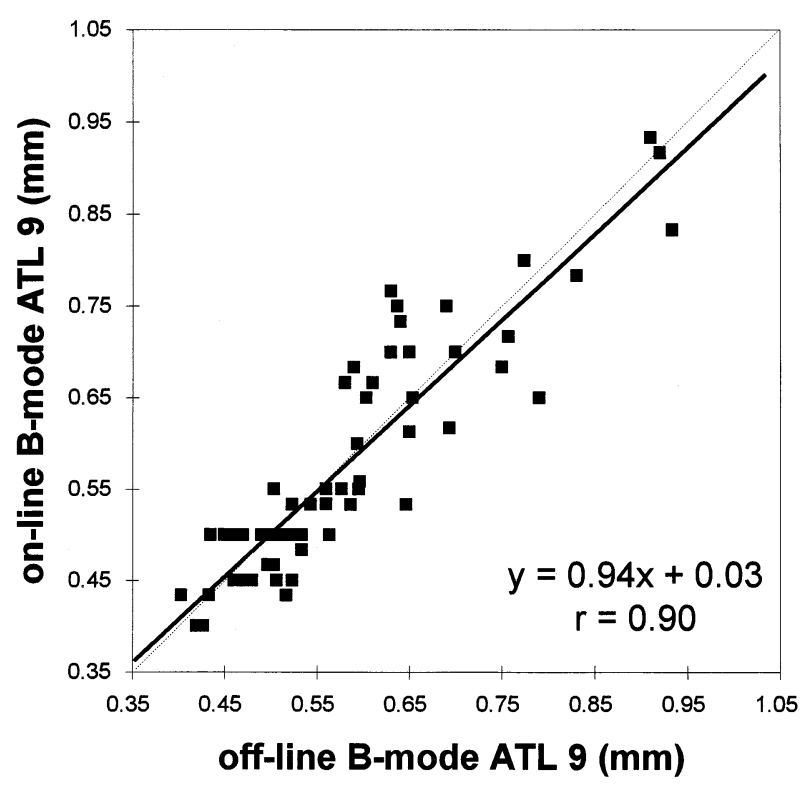

Fig. 8. Intima-media thickness (IMT) results obtained with the off-line manual B-mode IMT method as compared with the on-line manual B-mode IMT method. The regression line is displayed, as well as its equation and the $r$ value of the Spearman rank correlation coefficient. The dotted line represents the line of identity.

for longitudinal studies. Even when using the automated edge-detecting system of Selzer et al. (1994), the ultrasonographer still may influence the measurements, depending on the study design. It is important to note that both the success rate and the limits of agreement of IMT estimates in the present study are better in comparison to previous evaluation studies (Montauban van Swijndregt et al. 1996). This can be attributed partly to the subject population (healthy volunteers) studied.

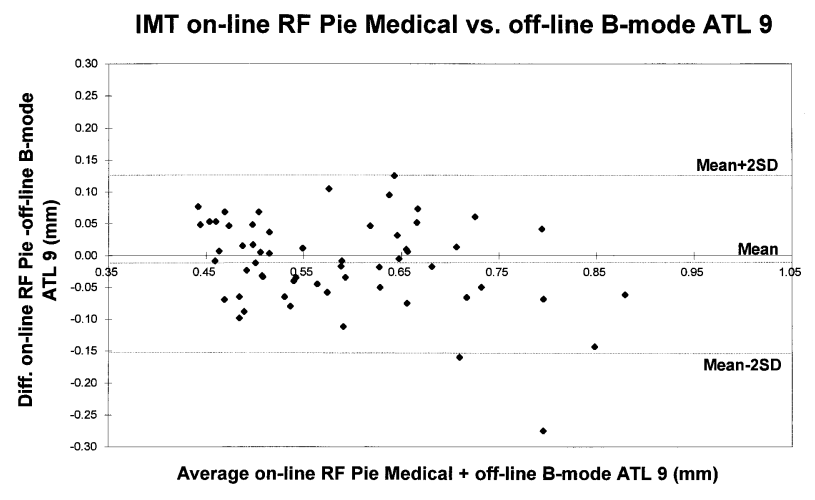

Fig. 9. Bland-Altman comparison between the off-line manual B-mode intima-media thickness (IMT) values and the on-line manual radio frequency (RF) IMT method. Depicted is the difference versus mean of both methods.

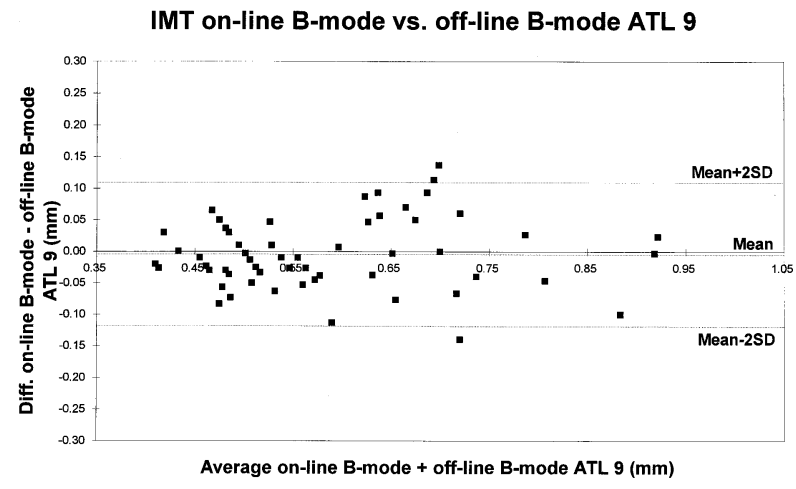

Fig. 10. Bland-Altman comparison between the off-line manual B-mode intima-media thickness (IMT) values and the on-line manual B-mode IMT method. Depicted is the difference versus mean of both methods.

The advantage of the off-line automated RF IMT method is that it not only is reliable, but it also is less time consuming and less user dependent than previously described methods. Another advantage of the RF method is that the same signal leads to simultaneous assessment of the local (relative) change in artery diameter over time and local IMT. With the parameters obtained and the local pulse pressure, the Young's modulus, the best parameter to describe the intrinsic elastic arterial properties, can be determined locally in an artery (Hoeks and Reneman 1995; Reneman et al. 1996). This is an asset in improving insight into arterial adaptation in processes such as ageing and hypertension.

We would like to emphasize the need to measure IMT locally, because of heterogeneity of intima thickening along an artery segment. This is especially important when studying the relation between IMT on the one hand and, for example, wall shear rate (Kornet et al. 1998), artery strain, or distension on the other locally. When interested in IMT as an indicator of a specific disorder, it may be more appropriate to average over a certain segment.

The off-line automated RF IMT results obtained with the Ultramark 9 and the Pie Medical Scanner 200 were comparable in outcome. This illustrates the insensitivity of the method for the ultrasound system characteristics.

The assessment of IMT averaged over the cardiac cycle instead of at the end-diastole, as formerly in the off-line manual B-mode IMT method, induces a systematic measurement error. The maximum bias in IMT will be on the order of $8 \%-10 \%$ in young volunteers and is dependent on the relative increase in diameter per heart beat (systolic-diastolic phase). This would explain an underestimation of $40-50 \mu \mathrm{m}$, for a mean IMT of 500 $\mu \mathrm{m}$ by the RF IMT method as compared to B-mode. In 
elderly volunteers, the relative increase in diameter decreases with age whereas the IMT increases, thereby leading approximately to the same absolute difference in IMT $( \pm 5 \%-6 \%$ of $750 \mu \mathrm{m}= \pm 37.5-45 \mu \mathrm{m})$. However, a smaller estimate of the off-line manual B-mode IMT was observed compared to the off-line automated RF method using the Ultramark 9 as well as the Pie Medical Scanner 200, especially in the older age group (Table 1). A possible explanation for the observed difference could be underestimation by the B-mode method due to either the delayed detection of the lumen-intima transition in $\mathrm{B}$-mode (absolute value rather than a relative one) or the earlier detection of the media-adventitia transition due to compression characteristics (Easton 1994). Differences between the reader of the images using the off-line manual B-mode in Rotterdam and the other methods performed in Maastricht also cannot be excluded.

In summary, we conclude that the off-line automated RF IMT method is a feasible method to assess IMT at the posterior wall of the common carotid artery as compared to the established off-line manual B-mode IMT method. One of the major advantages of this new automated procedure is that it is less user dependent and less time consuming than previously developed methods. Also, artery wall distensibility can be measured simultaneously at the same location, facilitating detailed study of the intrinsic wall characteristics.

\section{REFERENCES}

Bland JM, Altman DG. Statistical methods for assessing agreement between two methods of clinical measurement. Lancet 1986;i:307310.

Bond GM, Barnes RW, Riley WA, et al. The ARIC Study Group. High-resolution B-mode ultrasound scanning methods in the atherosclerosis risk in communities study (ARIC). J Neuroimag 1991; $1: 68-73$.

Bots ML, Mulder PG, Hofman A, van Es GA, Grobbee DE. Reproducibility of carotid vessel wall thickness measurements. The Rotterdam Study. J Clin Epidemiol 1994;47:921-930.

Chambless LE, Zhong MM, Arnett D, et al. Variability in B-mode ultrasound measurements in the atherosclerosis risk in communities (ARIC) study. Ultrasound Med Biol 1996;22:545-554.

Easton JD. Accuracy of high-resolution ultrasound imaging for quantitative assessment of early carotid atherosclerosis. Cerebrovasc Dis 1994:4:109-113

Hoeks APG, Reneman RS. Biophysical principles of vascular diagnosis. J Clin Ultrasound 1995;23:71-79. Hoeks APG, Brands PJ, Smeets FAM, Reneman RS. Assessment of the distensibility of superficial arteries. Ultrasound Med Biol 1990;16:121-128.

Hoeks APG, Willekes C, Boutouyrie P, et al. Automated detection of local artery wall thickness based on M-line signal processing. Ultrasound Med Biol 1997;23:1017-1023.
Kornet L, Lambregts J, Hoeks APG, Reneman RS. In the carotid artery differences in near wall shear rate within subjects are associated with different intima-media thicknesses. Arterioscler Thromb 1998; (In press).

Montauban van Swijndregt AD, The SH, Gussenhoven EJ, et al. An in vitro evaluation of the line pattern of the near and far walls of carotid arteries using B-mode ultrasound. Ultrasound Med Biol 1996;22:1007-1015.

O'Leary DH, Polak JF, Kronmal RA, et al. Thickening of the carotid wall. A marker for atherosclerosis in the elderly? Cardiovascular Health Study Collaborative Research Group. Stroke 1996;27:224231.

Persson J, Formgren J, Israelsson B, Berglund G. Ultrasound-determined intima-media thickness and atherosclerosis. Direct and indirect validation. Arterioscler Thromb 1994;14:261-264.

Persson J, Stavenow L, Wikstrand J, et al. Noninvasive quantification of atherosclerotic lesions. Reproducibility of ultrasonographic measurement of arterial wall thickness and plaque size. Arterioscler Thromb 1992;12:261-266.

Pignoli P. Ultrasound B-mode imaging for arterial wall thickness measurement. Atheroscler Rev 1984;12:177-184.

Pignoli P, Tremoli E, Poli A, Oreste P, Paoletti R. Intimal plus medial thickness of the arterial wall: A direct measurement with ultrasound imaging. Circulation 1986;74:1399-1406.

Reneman RS, Hoeks APG, Westerhof N. Non-invasive assessment of artery wall properties in humans-Methods and interpretations. J Vasc Invest 1996;2:53-64.

Riley WA, Barnes RW, Applegate WB, et al. Reproducibility of noninvasive ultrasonic measurement of carotid atherosclerosis. The Asymptomatic Carotid Artery Plaque Study. Stroke 1992;23:10621068.

Riley WA, Craven T, Romont A, Furberg CD. Assessment of temporal bias in longitudinal measurements of carotid intimal-medial thickness in the Asymptomatic Carotid Artery Progression Study (ACAPS). ACAPS Research Group. Ultrasound Med Biol 1996; 22:405-411.

Salonen R, Salonen JT. Progression of carotid atherosclerosis and its determinants: A population-based ultrasonography study. Atherosclerosis 1990;81:33-40.

Salonen JT, Salonen R. Ultrasonographically assessed carotid morphology and the risk of coronary heart disease. Arterioscler Thromb 1991;11:1245-1249.

Salonen R, Haapanen A, Salonen JT. Measurement of intima-media thickness of common carotid arteries with high-resolution B-mode ultrasonography: Inter- and intra-observer variability. Ultrasound Med Biol 1991;17:225-230.

Selzer RH, Hodis HN, Kwong FH, et al. Evaluation of computerized edge tracking for quantifying intima-media thickness of the common carotid artery from B-mode ultrasound images. Atherosclerosis $1994 ; 111: 1-11$.

Wendelhag I, Gustavsson T, Suurkala M, Berglund G, Wikstrand J. Ultrasound measurement of wall thickness in the carotid artery: Fundamental principles and description of a computerized analysing system. Clin Physiol 1991;11:565-577.

Wendelhag I, Liang Q, Gustavsson T, Wikstrand J. A new automated computerized analyzing system simplifies readings and reduces the variability in ultrasound measurement of intima-media thickness. Stroke 1997;28:2195-2200.

Wong M, Edelstein J, Wollman J, Bond MG. Ultrasonic-pathological comparison of the human arterial wall. Verification of intimamedia thickness. Arterioscler Thromb 1993;13:482-486. 